\title{
Simulation-Enhanced Atom Probe for Complete 3D Atomistic Imaging
}

M.P. Moody ${ }^{1}$, A.V. Ceguerra ${ }^{2}$, A .J. Breen ${ }^{2}$, B. Gault ${ }^{2}$, Xiang Yuan Cui ${ }^{2}$, Leigh T. Stephenson ${ }^{2}$, Ross K. W. Marceau ${ }^{3}$, Rebecca Powles ${ }^{2}$ and S.P. Ringer ${ }^{2}$.

1. Department of Materials, University of Oxford, Parks Road, Oxford, OX1 3PH, United Kingdom

2. Australian Centre for Microscopy and Microanalysis, and School of Aerospace, Mechanical and Mechatronic Engineering, The University of Sydney, NSW, 2006, Australia.

${ }^{3 .}$ Max-Planck-Institut für Eisenforschung GmbH, Max-Planck-Str. 1, 40237 Düsseldorf, Germany.

An analytical approach is developed blending the results of atom probe tomography with simulation to create a complete and lattice-based atomistic image of the original system. Although in one sense the information provided by APT is incomplete, in actuality the total amount of data generated by the technique is its exceptional strength. Hence, an almost overwhelming amount of statistical information is on offer for those prepared to delve, and it is this wealth of information upon which we build our platform to estimate the identities and positions of the atoms that we are missing.

In our approach, a crystalline specimen is analysed and a 3D atomic reconstruction of the original atomic configuration is generated. In the next step the average local chemical distribution within the reconstruction is characterised by measuring a set of short-range order (SRO) parameters $[1,2]$. The measured SRO provides a comprehensive description of the nanostructure present in the system. Next, upon application of statistical analyses, in many cases it is often observed that significant crystallographic information has been retained in the reconstruction. In turn, this information can be used to restore the data, atom-by-atom, to the perfect lattice configuration of the original specimen lattice rectification $[3,4]$. In the rectified data it is apparent that there are a significant number of vacant lattice sites. As a first approximation, it is assumed that this is due solely to limited detection efficiency. Atoms are then replaced into the APT data to filling the vacant sites. A Monte Carlo algorithm is then be utilised to swap atomic positions until the original SRO has been restored. The constraint is applied to this simulation that the original atoms are not moved during the Monte Carlo, only the positions of the restored atoms are swapped. Figure 1 highlights the main steps in this procedure as demonstrated for a simulated system. The result is a completed 3D lattice-based atomistic reconstruction of the original specimen.

\section{References:}

[1] A. V. Ceguerra et al., Physical Review B 82, (2010), p. 132201.

[2] A. V. Ceguerra et al., Acta Crystallographica Section A 68 (2012).

[3] M. P. Moody et al., Microscopy and Microanalysis 17, (2011) p. 226.

[4] F. Vurpillot et al., Ultramicroscopy 95, (2003) p. 223. 


\section{(a)}
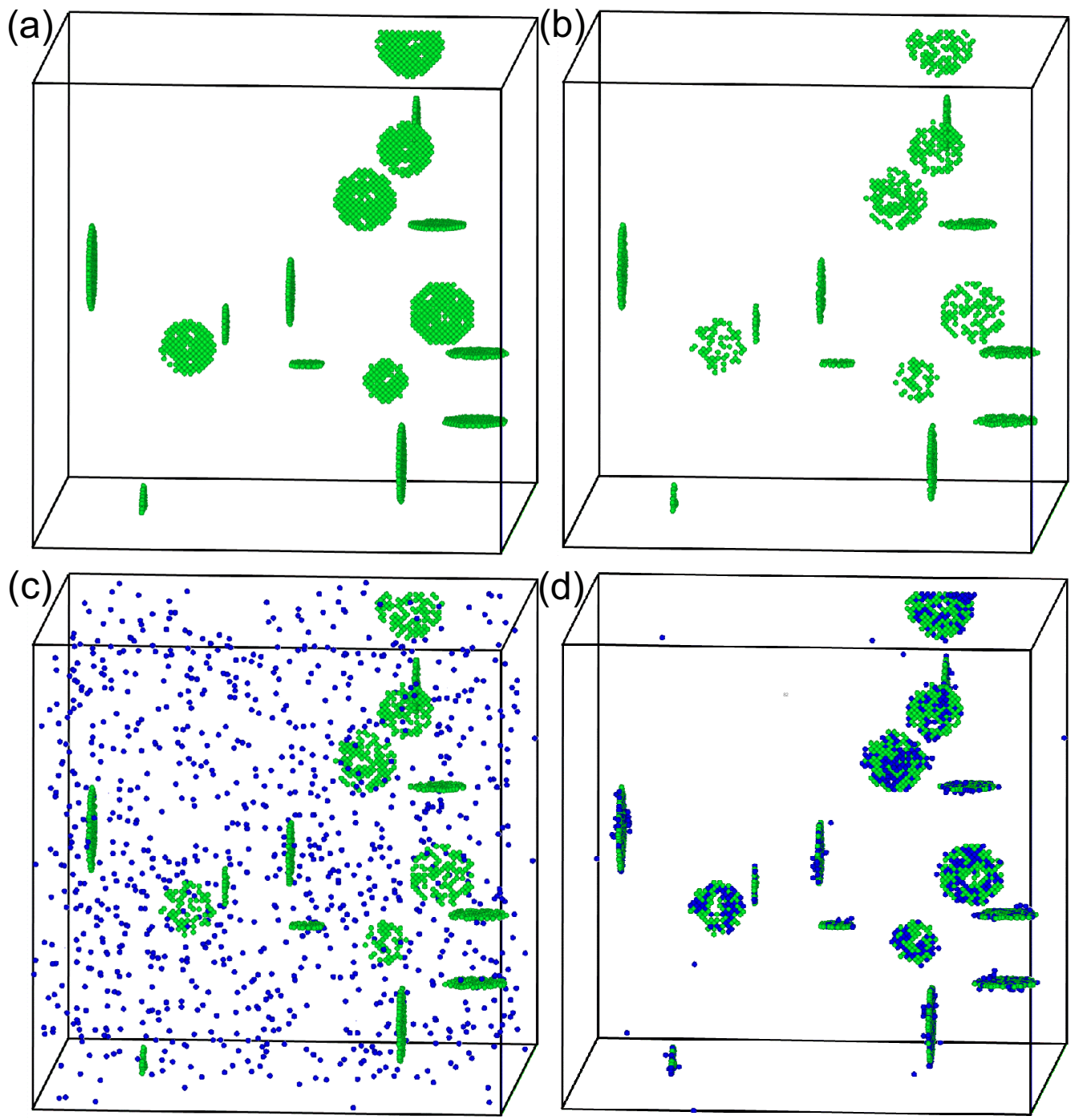

Figure 1. Predicting missing atoms in a simulated face-centred cubic lattice containing disc-shaped solute precipitates. Only solute atoms are shown. (a) Solute atoms by green spheres. All solute atoms are associated to single-layer disc shaped precipitates. (b) $43 \%$ of atoms have been removed to model the effect of APT detector efficiency. (c) Blue spheres are solute atoms that have been replaced at random into the vacant lattice sites. The overall composition of the system has been maintained. (d) The final system after distribution of the restored atoms is rearranged via MC simulation to return the original SRO. 